8th International Symposium on Electron Beam Ion Sources and Traps and

their Applications, BNL, Upton, NY, November 6-8, 2000

BNL -68992

\title{
Proposal for an EBIS-Based RHIC Preinjector
}

\author{
J.G. Alessi, E. Beebe, A.Kponou, A.Pikin, K. Prelec, \\ D. Raparia, J.Ritter, S.Y. Zhang
}

Brookhaven National Laboratory, Upton, NY 11973, USA

\begin{abstract}
A proposed new heavy ion preinjector for RHIC is described. The progress made at BNL on the development of an Electron Beam Ion Source (EBIS) has increased our confidence that one can build a preinjector meeting RHIC requirements using an EBIS producing intermediate charge state heavy ions. A new RFQ and Linac will be required to accelerate beams from this source to an energy sufficient for injection into the AGS Booster. These are both straightforward devices, very similar to ones already in operation at other laboratories. Injection into the Booster will occur at the same location as the existing heavy ion injection from the Tandem Van de Graaff.
\end{abstract}

\section{INTRODUCTION}

The present preinjector for heavy ions for AGS/RHIC uses the Tandem Van de Graaff, built around 1970. The beam is transported to the Booster synchrotron via an $860 \mathrm{~m}$ long line, as shown schematically in Figure 1. An alternative to this can be an Electron Beam Ion Source (EBIS), followed by a Radiofrequency Quadrupole (RFQ) accelerator, and a short Linac. This new preinjector offers improvements in both performance and operational simplicity.

The present scheme for filling RHIC uses one ion source pulse (and one Booster pulse) to fill one bunch in RHIC. Using Au as the most common example, for the required $10^{9}$ ions per bunch in RHIC, and with a transfer efficiency of about $50 \%$ (including stripping after the Booster) from Booster to AGS (and RHIC), one needs to achieve $2 \times 10^{9}$ Au ions per pulse extracted from the Booster.

Present heavy ion injection into the Booster from the Tandem Van de Graaff starts with a sputter negative ion source on a $150 \mathrm{kV}$ high voltage platform, which can deliver approximately $200 \mu \mathrm{A}$ of $\mathrm{Au}^{-}$in $500 \mu$ s pulses. The beam is then accelerated to the Tandem's $14 \mathrm{MV}$ terminal, where ions are stripped by passage through a 2 $\mu \mathrm{g} / \mathrm{cm}^{2}$ carbon foil, and then accelerated to ground potential, where the total current (all charge states) is $\sim 1 \mathrm{emA}$, with approximately $20 \%$ of that being in the desired $12+$ charge state. At ground, the beam then passes through a second carbon stripper foil, with $\sim 70 \mathrm{e} \mu \mathrm{A}$ in the desired $\mathrm{Au}^{32+}$ charge state, with an energy of about 0.92 $\mathrm{MeV} / \mathrm{amu}$. This beam is transported $860 \mathrm{~m}$ to the Booster, where it is injected over 35 turns, with a capture efficiency of about $50 \%$. 


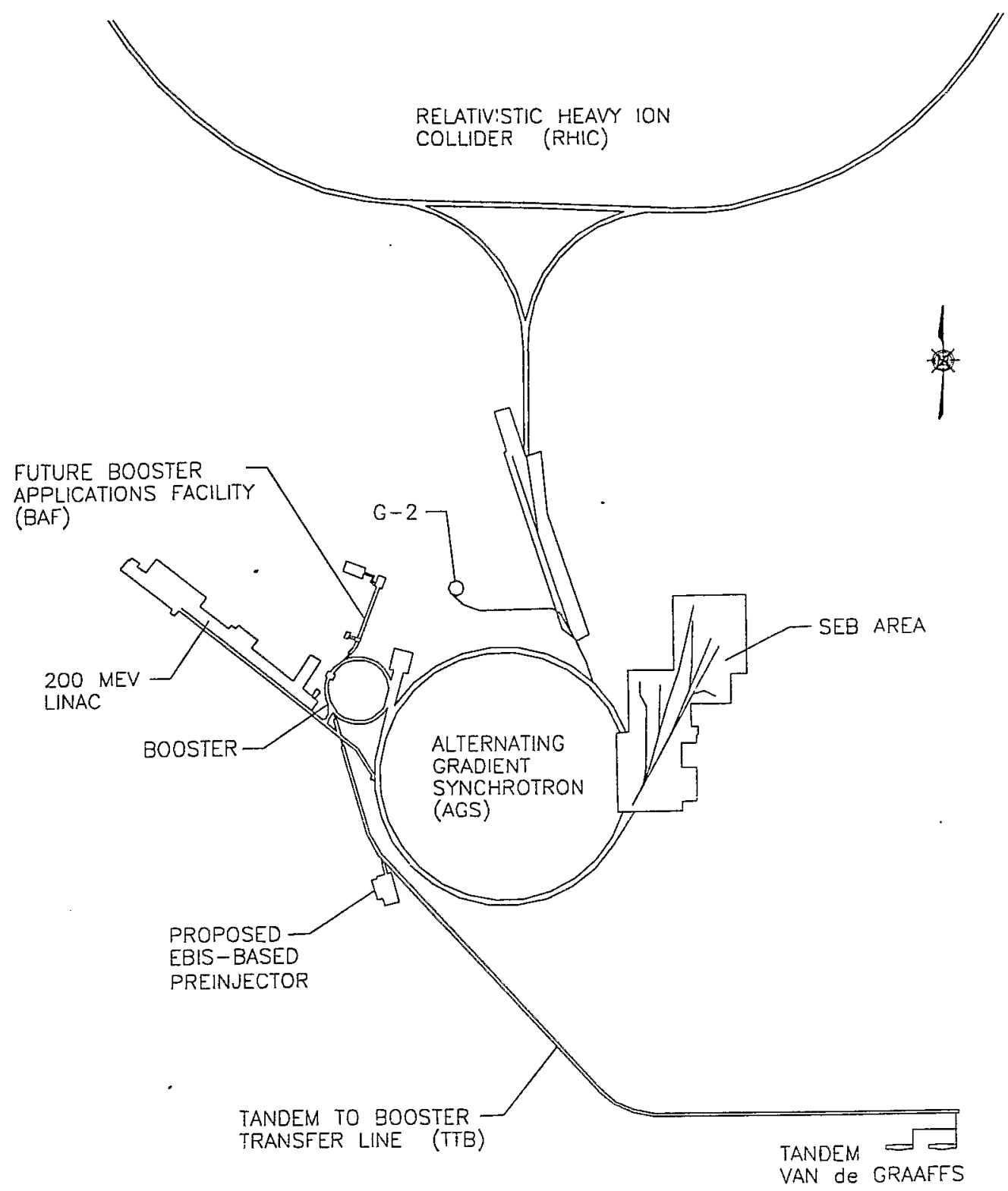

FIGURE 1. Schematic view of the present Tandem injection and future EBIS injection lines.

An attractive alternative scheme is to produce, directly from an ion source, the charge state desired for Booster injection. This eliminates the inefficiencies due to stripping, and makes the initial preacceleration more efficient. In addition, Booster injection is more efficient if one can inject over fewer turns, so it is also desirable for the source to produce shorter pulses of higher currents. Some of the parameters required from a new preinjector are listed as follows:

1. Intensity at Booster injection: $2.7 \times 10^{9} \mathrm{Au}^{32+}$ ions per pulse. Sufficient to achieve $1 \times 10^{9}$ ions per bunch in RHIC.

2. Pulse width: variable, $\mathbf{1 0 - 4 0 \mu s . ~ T h i s ~ a l l o w s ~ 1 - 4 ~ t u r n ~ i n j e c t i o n ~ i n t o ~ t h e ~ B o o s t e r . ~}$ This simplifies the injection, and should greatly reduce the sensitivity to small 
beam losses at injection, which may cause a pressure bump resulting in further beam loss.

3. Repetition rate: $\mathbf{5 - 1 0} \mathbf{H z}$. This keeps overall RHIC fill times to only a few minutes

4. Injection energy: $2 \mathrm{MeV} / \mathbf{a m u}$. Present Tandem injection is at $0.92 \mathrm{MeV} / \mathrm{amu}$ for $\mathrm{Au}$. At this energy, there is a significant beam loss due to electron capture during Booster injection. By raising the injection energy to $2 \mathrm{MeV} / \mathrm{amu}$, the capture cross section is reduced by a factor of 20-40. In addition, the higher energy reduces the space charge tune shift at injection, which might be important at these higher peak currents. At even higher injection energies one would approach the voltage limit of the inflector, and losses due to ionization would begin to become important.

5. $q / \mathbf{m}: \mathbf{0 . 1 6}$ or greater. This ratio equals that presently delivered for $A u$ from the Tandem. For lighter ions, higher $\mathrm{q} / \mathrm{m}$ is required $\left(\mathrm{Si}^{14+}, \mathrm{Fe}^{21+}\right.$ ) to achieve the desired Booster output energy, within rigidity constraints in the Booster and extraction transport.

6. Emittance (90\%, unnormalized): $11 \pi \mathrm{mm}$ mrad or less. Matches acceptance of the inflector with minimal losses. This emittance is acceptable for the 1-4 turn injection (if one were to inject over 10's of turns, as with the Tandem, the emittance requirement is stricter).

7. $\mathrm{dp} / \mathrm{p}: \mathbf{0 . 0 5 \%}$ or less.

These parameters can be achieved with an EBIS source, followed by an RFQ and short Linac. A schematic of the injection scheme with the new injector is shown in Fig. 2.

\section{FEATURES AND ADVANTAGES OF THE NEW PREINJECTOR}

Along with the present Tandem, a possible location for the proposed preinjector is shown in Fig. 1. The new beamline joins the existing Tandem-to-Booster (TTB) line at the first of two $13^{\circ}$ dipoles. After this first bend, one can separate out unwanted charge states in the EBIS preinjector beam. Existing quadrupoles are left at their present locations, so the Tandem injection remains unchanged. Additional quadrupoles are added after this merging of the lines to accommodate the EBIS injection.

Linac-based preinjectors are presently used at most accelerator and collider facilities with the exception of RHIC, where the required gold beam intensities could only be met with a Tandem until the recent EBIS development. The high reliability and flexibility of a new Linac-based preinjector will be an essential component for the long-term success of the RHIC facility. The Linac-based preinjector offers the following advantages:

- The RFQ and Linac are a simpler, more robust technology, which will require less maintenance than the Tandem. This is similar to our very favorable experience of replacing a large electrostatic device, the Cockcroft -Walton preaccelerator, with a compact RFQ accelerator for $\mathrm{H}^{-}$ions in the $200 \mathrm{MeV}$ Linac. 


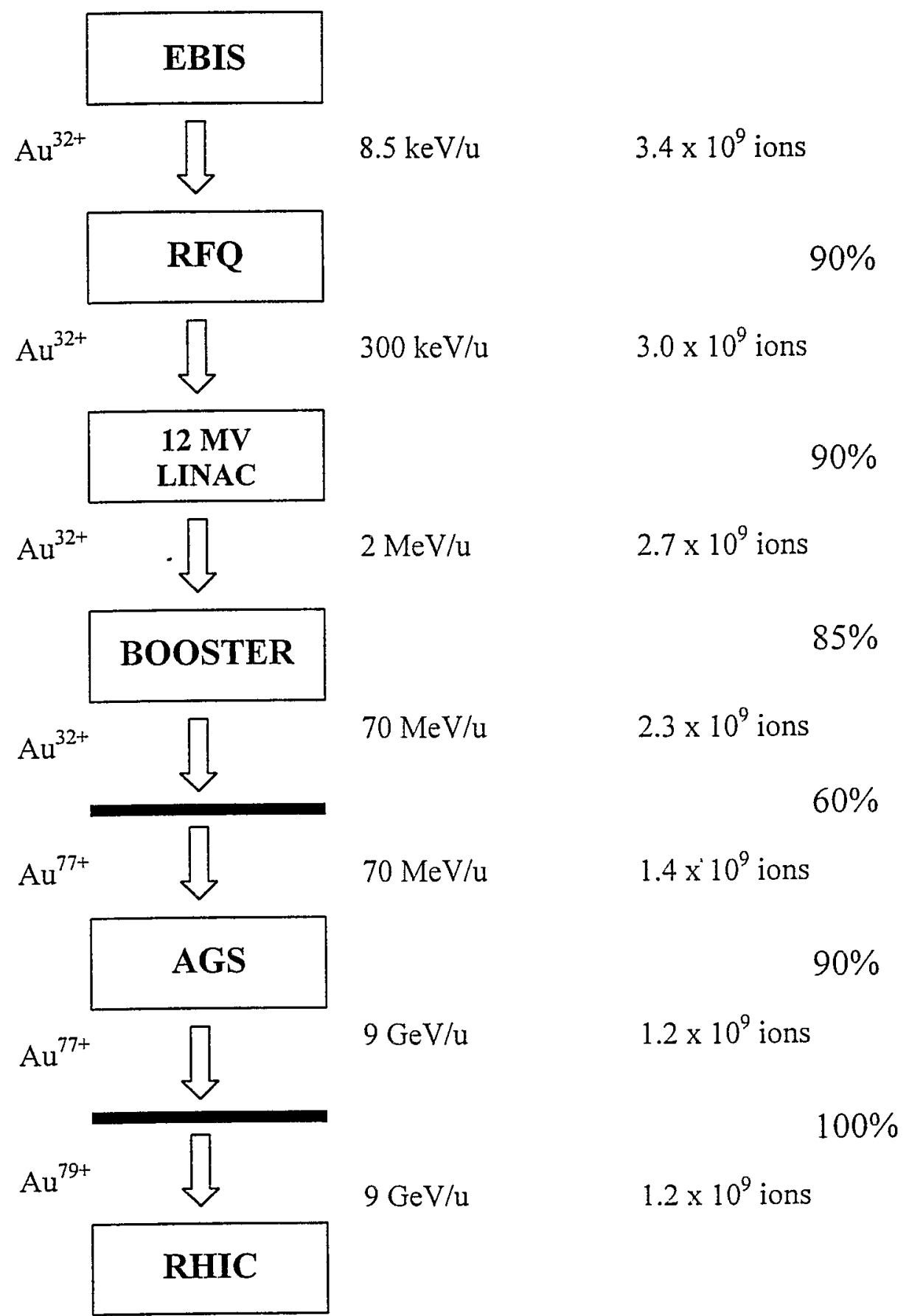

FIGURE 2. Schematic of EBIS-based injection, showing ions per pulse, and efficiencies at various stages.

- The Tandem requires stripping foils at two locations. Although foil lifetime should become less of an issue as RHIC beam storage times increase, the energy spread in the Tandem beam increases as the foils age (thicken). The EBIS requires no stripping before the Booster. 
- The $860 \mathrm{~m}$ long Tandem-to-Booster transport is difficult to tune, especially when changing the species. The new line would be only about $160 \mathrm{~m}$ long, and would use a more stable lattice.

- The EBIS will inject only 1-4 turns, as opposed to 30-40 from Tandem, so injection will be much easier.

- The higher Booster injection energy will reduce losses at injection.

- Tandem species are limited to ions starting as negatives, while the EBIS can produce all ions.

- The EBIS can switch species very quickly, for filling RHIC with two different ions, or for fast switching between RHIC and the Booster Application Facility (BAF). Fast switching with the Tandem requires the use of the two BNL Tandems.

With the long-term commitment to heavy ions at BNL for RHIC, and increasing demands for different species and delivery of beams to different users, the EBIS preinjector will enhance capabilities significantly. Undoubtedly, additional unexpected impacts of the EBIS preinjector will appear as one gains experience and explores the new parameter space it will present. Even with a new preinjector, one can imagine that the Tandem will continue to play an important role. Multiple injectors are not uncommon at heavy ion facilities since the preinjectors are a relatively lowcost, high impact part of a facility.

\section{THE EBIS SOURCE}

An EBIS delivers pulses having a constant total positive charge, and one has control over the ion pulse width by controlling the release of the trap voltage. Ions can be extracted in short pulses of high current, which is desirable for synchrotron injection. With the properties of an EBIS being well understood, one can arrive at design parameters for an EBIS meeting RHIC requirements. These parameters are given in Table 1. While this combination of parameters is not unique, based on past and present experience we feel that they represent the most straightforward path to the design goals. Also given in the table are some of the presently achieved parameters from the BNL EBIS test stand (EBTS).

TABLE 1. EBIS Parameters

\begin{tabular}{|l|c|c|}
\hline \multicolumn{1}{|c|}{ Parameter } & RHIC EBIS & $\begin{array}{c}\text { EBTS } \\
\text { (achieved to date) }\end{array}$ \\
\hline e-beam current & $10 \mathrm{~A}$ & $10 \mathrm{~A}$ \\
\hline e-beam energy & $20 \mathrm{keV}$ & $20 \mathrm{keV}$ \\
\hline e-beam density & $\sim 575 \mathrm{~A} / \mathrm{cm}^{2}$ & $>575 \mathrm{~A} / \mathrm{cm}^{2}$ \\
\hline Ion trap length & $1.5 \mathrm{~m}$ & $0.7 \mathrm{~m}$ \\
\hline Trap capacity (charges) & $1.1 \times 10^{12}$ & $5.1 \times 10^{11}(10 \mathrm{~A})$ \\
\hline Yield positive charges & $5.5 \times 10^{11}$ & $1.9 \times 10^{11}(\mathrm{Xe}, 7 \mathrm{~A})$ \\
& & $2.8 \times 10^{11}(\mathrm{Bkgd}$ gas ions, $7 \mathrm{~A})$ \\
\hline Yield $\mathrm{Au}^{32+}$, design value & $3.4 \times 10^{9}$ ions/pulse & \\
\hline Yield $\mathrm{U}^{45}$, design value & $2.4 \times 10^{9}$ ions/pulse & \\
\hline
\end{tabular}


The electron beam neutralization efficiency for RHIC EBIS is assumed to be $50 \%$, a value that has been exceeded on our EBTS, as well as in many other EBISs. The yield in the desired charge state is assumed to be $20 \%$, again a value which is frequently achieved in EBISs.

We have also considered other sources for the production of pulsed currents of intermediate charge state ions for synchrotron injection - specifically the Electron Cyclotron Resonance (ECR) ion source, and the Laser Ion Source (LIS). These sources have a more difficult time achieving the desired charge state, so while with an EBIS one is using the peak charge state from a narrow distribution, with the ECR and LIS one is typically using a charge state on the tail of a much broader distribution. Thus, for the same current in the desired charge state, one has to deal with much higher total extracted currents, and their accompanying problems of transport and matching into the RFQ. For example, $3.4 \times 10^{9} \mathrm{Au}^{32+}$ ions in a $10 \mu$ sulse corresponds to a $\mathrm{Au}^{32+}$ current of $1.7 \mathrm{~mA}$. The total extracted current from the EBIS will be $8.5 \mathrm{~mA}$, assuming $20 \%$ in the desired charge state. For the same $\mathrm{Au}^{32+}$ current, total current from an ECR or LIS could be up to 10 times higher (this is considerably above what an ECR has achieved). If one goes to a lower charge state from the ion source, then in addition to the accelerator becoming longer, the total current required becomes even higher because an additional stripping would be required after acceleration.

Both sources also have some limitations in the ions that can be produced, the LIS requiring high melting point solids, and the ECR having difficulty producing ions from high melting point solids. The LIS has additional obstacles, such as larger emittance due to a large energy spread, target erosion and coating of mirrors, state of the art laser required, and large pulse-to-pulse fluctuations in beam current.

Unlike these two sources, an EBIS can easily produce any type ions - from gas, metals, etc., and can quickly switch species (even pulse-to-pulse) without a memory effect. One can easily control the width of the extracted pulse. Scaling laws for EBIS are well understood, and the source is reliable, with excellent pulse-to-pulse stability.

\section{RESULTS OF THE EBIS TEST STAND}

The requirements for the RHIC EBIS were given in Table 1. These parameters were considerably beyond the previous state of the art, since most EBIS sources were designed for atomic physics applications, where much lower intensities of very high charge state ions were usually desired. The most recent phase of the BNL EBIS development program has been to demonstrate that an EBIS capable of meeting the RHIC requirements can be built. Our approach has been to construct a full power, close to $1 / 2$ length prototype, show that each subsystem can work, demonstrate ion production and extraction in expected quantities, and finally demonstrate the production of heavy ions. with $\mathrm{q} / \mathrm{A} \sim 0.16$ centered in a narrow charge state distribution. With this EBIS Test Stand we have been able to develop many of the relevant technologies, and study the physics aspects of a high intensity EBIS. A number of issues have been addressed, among them the technology of high current electron beam 
formation and launching, development of primary ion injection into the trap, the study of ion formation in and loss from a high current electron beam, the study of fast ion extraction, and the development of appropriate source controls and diagnostics.

Details of the EBTS design and experimental results are presented in another paper in these Proceedings [1]. Only some of the key achievements will be mentioned in the following discussion.

\section{Demonstration of High Current Electron Beam Formation and Propagation}

The design of the electron gun was of crucial importance not only because of the requirement for such a high current, but also because of the need for a flexible control of the electron beam parameters. After performing an extensive study of different electron gun geometries it was decided to adopt a coaxial diode with magnetic insulation, positioned in the field of a separate solenoid. The gun was designed and fabricated at the Budker Institute of Nuclear Physics, Novosibirsk [2].

The performance of this novel electron gun has been excellent. It has provided very stable operation over a wide range of gun operating parameters, with very satisfactory lifetime and reliability. With this gun we have reached our design goal, and propagated a $10 \mathrm{~A}$ electron beam through the EBIS solenoid to the collector, with very low beam loss $(<0.5 \%)$, in $10 \mathrm{~ms}$ pulses.

\section{Extraction of Ions from the EBTS Trap}

Our design goal of extraction of a total ion charge corresponding to $50 \%$ of the electron beam space charge has consistently been met or exceeded. Trapping and ionization of background gas ions was the most straightforward first test of operation, since the EBTS vacuum was initially worse than desired due to a collector cooling water leak into the vacuum system. Subsequently, after repairing the leak, operation with continuous injection of Xe gas into the EBTS demonstrated good operation as well. Table 2 shows some of the ion yields under various operating conditions. The RHIC EBIS requires a yield of $5 \times 10^{11}$ positive charges/pulse, at $10 \mathrm{~A}$ but at twice the trap length of EBTS.

TABLE 2. Ion yields from EBTS

\begin{tabular}{|l|c|c|c|}
\hline \multicolumn{1}{|c|}{ Ion } & Electron current & Ion yield charges/pulse & Neutralization \\
\hline Background gas & $7.0 \mathrm{~A}$ & $2.8 \times 10^{11}$ & $85 \%$ \\
\hline Xenon & $7.0 \mathrm{~A}$ & $1.9 \times 10^{11}$ & $55 \%$ \\
\hline
\end{tabular}

We can not yet measure ion yields at the full electron beam current. Practical limitations to the voltages that can be applied to the trapping electrodes prevent trap formation when one has a deeper potential well from the higher electron beam currents. In the future, planned design modifications will allow extraction of ions from a $10 \mathrm{~A}$ electron beam. 


\section{Fast Extraction of Ions from the EBTS Trap}

For 1-4 turn injection into the Booster, the extracted ion pulse should be 10-40 $\mu \mathrm{s}$ long. Figure 3 is a $10 \mu \mathrm{s}$ FWM ion pulse extracted from EBTS, demonstrating that this requirement can be met at a high electron beam current. This result was achieved by raising the voltage of the trap region above the level of the barrier electrode, with an additional voltage tilt in the trap produced via a resistor/capacitor network. In a RHIC EBIS, with straightforward programmable control of electrode voltages, the shape and duration of the pulse will be controllable. The peak current is $3.3 \mathrm{~mA}$. While this result shows ions produced from background gas, this fast extraction can be similarly achieved for any ions.

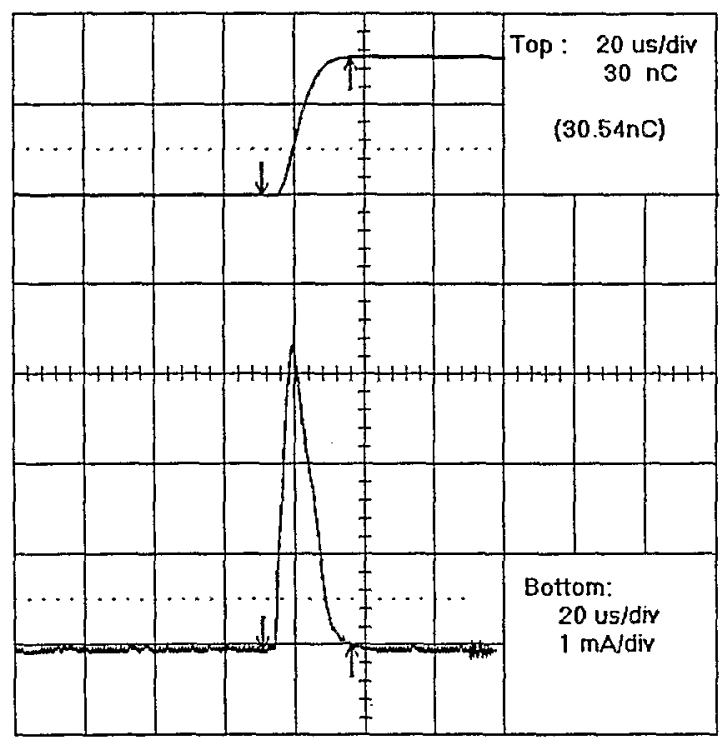

FIGURE 3. Bottom trace: $3.3 \mathrm{~mA}, 10 \mu \mathrm{s}$ FWH ion pulse extracted from the EBIS with a $6 \mathrm{~A}, 17.6 \mathrm{kV}$ electron beam and $10 \mathrm{~ms}$ confinement time. Top trace: Integral of bottom trace $(30.5 \mathrm{nC}$ total charge).

\section{Measurement of Charge State Distribution of Ions from EBTS}

Charge state distributions were measured with a time-of-flight spectrometer located $1.5 \mathrm{~m}$ from the ion extraction electrode. Distributions were measured using both $\mathrm{Ar}$ and Xe gas injection. The dependence of the peak charge state on $j \tau$, the product of electron beam current density and confinement time, is well understood. If one uses the measured charge state distribution to determine $j \tau$ and then scales to a $10 \mathrm{~A}$ electron beam operation, it will be sufficient for the production $\mathrm{Au}^{32+}$ or $\mathrm{U}^{45+}$ within $100 \mathrm{~ms}$ with a factor of 2 safety margin.

A lower charge state tail was measured, which is typical of continuous gas injection. Narrower charge state distributions have been demonstrated on EBIS' such as those in Stockholm and Saclay using injection of singly charged ions from an external source into the trap. In the EBTS, we have demonstrated this as well, by injecting ions from an external $\mathrm{Cs}^{1+}$ source. The intensity of the external ion source presently limits our 
extracted Cs ion yield. More intense sources providing singly charged ions for a broad range of species are available commercially; also the optics of the present external source can be improved considerably, resulting in more primary ions injected into the EBIS.

\section{Summary of EBTS Performance}

- We have achieved the design goal of transporting a $10 \mathrm{~A}$ electron beam through the $0.7 \mathrm{~m}$ EBTS trap with low losses.

- The extraction of ion pulses of $2.8 \times 10^{11}$ charges with a 7A electron beam is less than a factor of 2 below the RHIC requirement for charge, and has demonstrated proper operation of an EBIS at high currents.

- Measured charge state distributions for Xe and Cs ions are consistent with the expected values based on the electron current density and ion confinement time.

- Ions from an auxiliary Cs ion source were successfully injected into the trap of EBTS with a good efficiency. Studies will continue to optimize ion and gas injection, beam extraction and transport from the EBTS.

- To date, all results of the EBTS have agreed with EBIS scaling laws, and continue to confirm the parameters for a RHIC EBIS that were presented approximately 10 years ago.

Practical constraints rather than physics issues have limited performance of the EBTS, and therefore, while the test stand will benefit from further design optimization, we are now confident that an EBIS can be scaled to meet RHIC requirements.

\section{FEATURES OF AN EBIS FOR RHIC}

Our experience so far in the operation of the EBTS has confirmed the validity of our approach to the design of the RHIC EBIS. New features we plan to incorporate into the final EBIS will make the final EBIS more robust. A schematic of the RHIC EBIS is shown in Fig. 4. Presented below is our present concept for several key EBIS components. (Details may still change as a result of our ongoing EBTS R\&D).

\section{Electron Gun}

As has been mentioned previously, the required intensity of $\sim 3 \times 10^{9}$ of $\mathrm{Au}^{32+}$ ions per pulse can be provided with a trap containing $\sim 1 \times 10^{12}$ electrons. If the trap is $1.5 \mathrm{~m}$ long and energy of electrons is $20 \mathrm{keV}$, the electron beam current should be $10 \mathrm{~A}$. The microperveance of this electron beam is 3.5. There are several strict requirements to the electron beam, such as having the ability to be decelerated strongly in the strong magnetic field and in the collector region, and having the ability to be operated over a wide parameter range. The existing electron gun with convex cathode and pure magnetic compression of the electron beam has proven to satisfy all our requirements. 


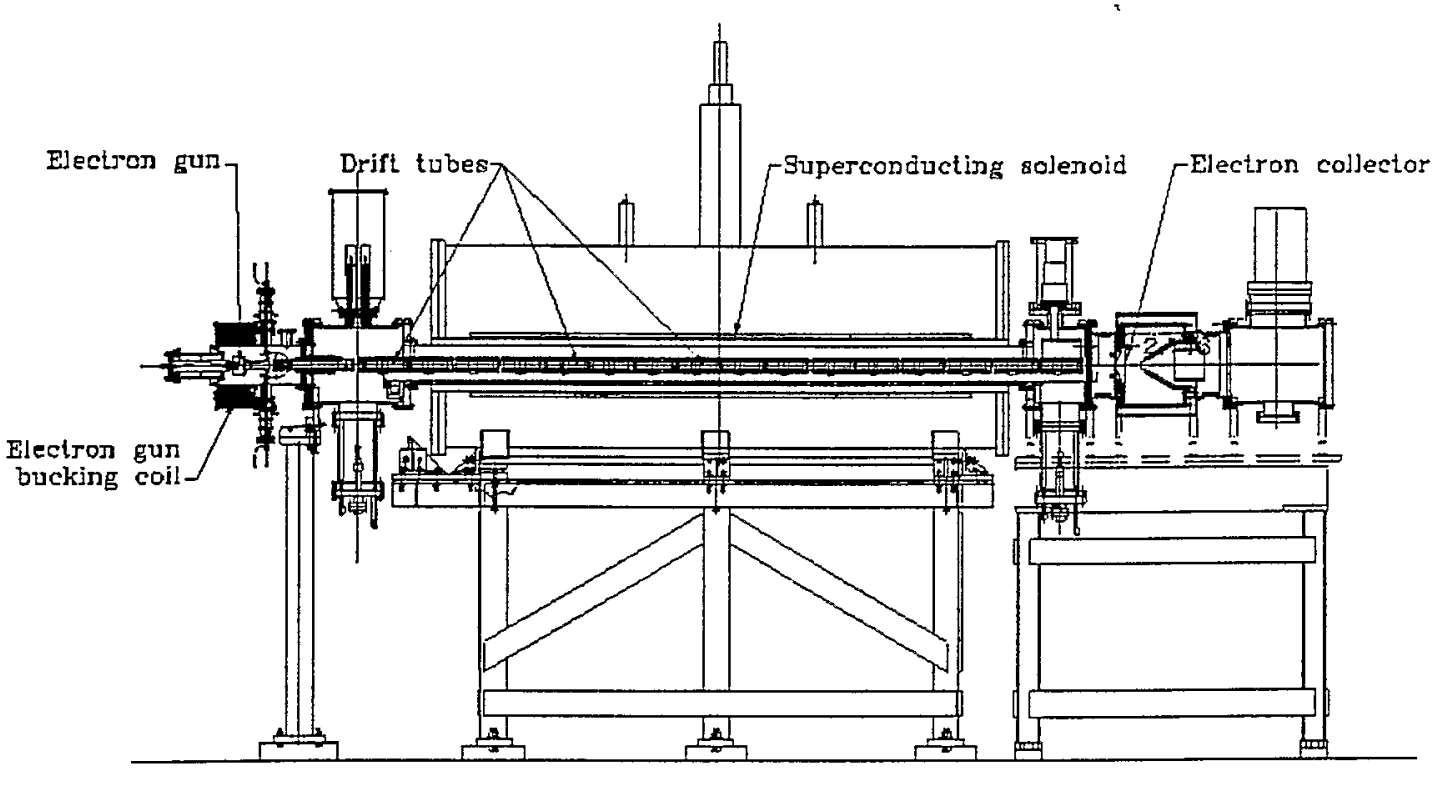

FIGURE 4. Schematic of the proposed EBIS for RHIC.

The existing electron gun can generate an electron current of $10 \mathrm{~A}$ for 1000 hours. After that the quality of electron beam becomes unsatisfactory because of deterioration of the cathode unit, and a replacement of the cathode is required. The existing unit meets our requirements, and it will be used for the RHIC EBIS as well. However, to have a comfortable safety factor and a reserve for a possible future increase of the ion beam intensity, it would be advantageous to have an additional electron gun which is capable of generating an electron beam with a higher current, for example $15 \mathrm{~A}$. It is probably not feasible to increase the current by boosting emission from the existing cathode, because the lifetime of the gun would become unacceptably short. Instead, we propose to order a second electron gun based on the same principles, but with a larger cathode. An electron gun with cathode diameter $10.2 \mathrm{~mm}$ (instead of the present $8.3 \mathrm{~mm}$ ) will be able to generate a $15 \mathrm{~A}$ electron beam with the same current density as the existing cathode $\left(18.5 \mathrm{~A} / \mathrm{cm}^{2}\right)$. The cathode will have the same lifetime if it is made from a single crystal $\mathrm{LaB}_{6}$, as it is now. We are presently discussing the design of this new gun with scientists at BINP, and their institute could again do detailed design and fabrication, as was the case for our $10 \mathrm{~A}$ gun.

\section{Electron Collector}

The main objective of the new electron collector (EC) for the RHIC EBIS is to increase its capacity to dissipate power, compared with the existing EC on EBTS. The new EC should be designed to dissipate a power of $230 \mathrm{~kW}$, which is 4.5 times higher than the existing EC, and 2.3 times higher than our expected load of $100 \mathrm{~kW}$. In fact, the new EC will be able to dissipate the full power of a $15 \mathrm{~A}$ electron beam in DC mode. To increase the capacity of the EC, three approaches will be used - the peak power density at the cooling water channels will be reduced relative to the inner 
surface power density, the heat exchange by cooling water will be increased, and the pressure of the cooling water will be increased.

To reduce the maximum power density on the surface-water interface, the longitudinal distribution of the electron beam on this surface will be made more homogeneous than in the existing EC by optimizing the shape of the magnetic field. The total area of the cylindrical water-cooled surface of EC will also be increased, with the new collector having an inner diameter of $\sim 30 \mathrm{~cm}$ and a length of $\sim 24 \mathrm{~cm}$. The ratio of surface area of cooling channels to the area of inner EC cylindrical surface will be increased. The flow rate of cooling water will be 4 GPM through a single channel, two times higher than in the existing EBTS. Raising the pressure of the cooling water to $25 \mathrm{bar}$ increases its boiling temperature to $200 \mathrm{C}$, making possible a heat exchange without creating a vapor sheath on the surface for a local power density up to $700 \mathrm{~W} / \mathrm{cm}^{2}$.

\section{Superconducting Solenoid}

Table 3 shows the parameters of the new solenoid, as well as the parameters of the existing EBTS solenoid. The coil for the new solenoid will be split into three independently adjustable regions ( $25 \% / 50 \% / 25 \%$ of length), to allow some flexibility in the shaping of the axial field. The diameter of the warm bore of the solenoid may be increased to facilitate pumping, and to provide more space to reduce the likelihood of high voltage breakdowns.

TABLE 3. Required parameters of the superconducting solenoid and present EBTS solenoid parameters

\begin{tabular}{|l|c|c|}
\hline & RHIC EBIS & EBTS \\
\hline Maximum magnet field & $5 \mathrm{~T}$ & $5 \mathrm{~T}$ (tested to 5.5) \\
\hline Inner diameter of the warm bore & $\begin{array}{c}204 \mathrm{~mm} \\
\text { (clear for 8" flange) }\end{array}$ & $\begin{array}{c}155 \mathrm{~mm} \\
\text { (clear for 6") }\end{array}$ \\
\hline Total length of solenoid & $2000 \mathrm{~mm}$ & $1000 \mathrm{~mm}$ \\
\hline Homogeneity over region $1300 \mathrm{~mm} \times 10 \mathrm{~mm}$ & $0.25 \%$ & $0.25 \%$ \\
\hline $\begin{array}{l}\text { Maximum radial shift of magnet field axis } \\
\text { over full length of the magnet (documented) }\end{array}$ & $0.2 \mathrm{~mm}$ & $0.2 \mathrm{~mm}$ \\
\hline $\begin{array}{l}\text { Maximum radial deviation of position of } \\
\text { solenoid axis from the position of warm } \\
\text { bore axis }\end{array}$ & $0.2 \mathrm{~mm}$ & $0.2 \mathrm{~mm}$ \\
\hline $\begin{array}{l}\text { Decay rate of magnet field in coils of } \\
\text { solenoid, operating with current leads } \\
\text { removed. }\end{array}$ & $1 \times 10^{-6}$ per hour & $1 \times 10^{-5} \mathrm{per}$ hour \\
\hline Length of vacuum jacket & $\sim 2300 \mathrm{~mm}$ & $1300 \mathrm{~mm}$ \\
\hline Period between liquid helium refills & 30 days & 23 days \\
\hline Period between liquid nitrogen refills & 10 days & 12 days \\
\hline
\end{tabular}




\section{TRANSPORT AND ACCELERATION}

Some parameters of the preinjector are given in Table 4. The details of the subsystems are given in the following sections. A layout of the preinjector is shown in Fig. 5.

TABLE 4. Beam parameters of the proposed preinjector

\begin{tabular}{|c|c|c|c|}
\hline \multicolumn{4}{|l|}{ EBIS } \\
\hline & $\begin{array}{l}\text { Output (single } \\
\text { charge state) }\end{array}$ & $1.1 \times 10^{11}$ & Charges \\
\hline & Ion output $\left(\mathrm{Au}^{32+}\right)$ & $3.4 \times 10^{9}$ & particles/pulse \\
\hline & Pulse width & $10-40$ & $\mu \mathrm{S}$ \\
\hline & Max rep rate & 10 & $\mathrm{~Hz}$ \\
\hline & $\begin{array}{l}\text { Beam current } \\
\text { (single charge state) }\end{array}$ & $1.7-0.42$ & $\mathrm{~mA}$ \\
\hline & Output energy & 8.5 & $\mathrm{keV} / \mathrm{amu}$ \\
\hline & Output emittance & 0.35 & $\pi \mathrm{mm} \mathrm{mrad}$, norm, $90 \%$ \\
\hline \multicolumn{4}{|l|}{ RFQ } \\
\hline & $\mathrm{Q} / \mathrm{m}$ & $0.16-0.5$ & \\
\hline & Input energy & 8.5 & $\mathrm{keV} / \mathrm{amu}$ \\
\hline & Output energy & 300 & $\mathrm{keV} / \mathrm{amu}$ \\
\hline \multicolumn{4}{|c|}{ IH Linac } \\
\hline & $\mathrm{Q} / \mathrm{m}$ & $0.16-0.5$ & \\
\hline & Input energy & 300 & $\mathrm{keV} / \mathrm{amu}$ \\
\hline & Output energy & 2000 & $\mathrm{keV} / \mathrm{amu}$ \\
\hline \multicolumn{4}{|c|}{ Injection } \\
\hline & \# of turns injected & $1-4$ & \\
\hline
\end{tabular}

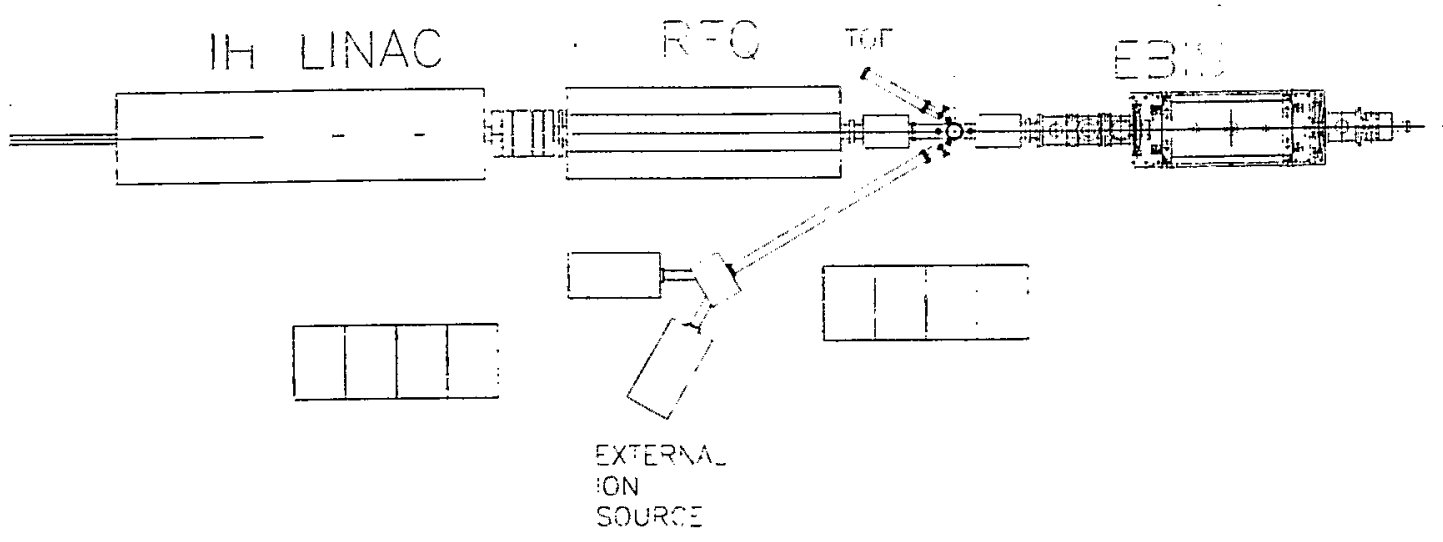

FIGURE 5. Conceptual layout of the preinjector 


\section{Low Energy Beam Transport}

The Low Energy Beam Transport (LEBT) transports the beam from the EBIS and matches it to the RFQ. The LEBT is 1.4 meters long and consists of two solenoid magnets for transverse matching, two sets of transverse steerers, and a Y-chamber in the middle of the line. One arm of this chamber allows ions from an external ion source to be injected into the EBIS trap. In the second arm extracted ions can be deflected into a time-of-flight diagnostic.

The bearn from the EBIS source has to be matched into the RFQ, which needs a small, highly convergent beam. The beam out of EBIS is symmetric in $x$ and $y$, as is the RFQ acceptance, so one needs control of only two degrees of freedom for matching. Figure 6 shows the beam envelopes along the LEBT (output from TRACE code [3]). The magnetic fields required for the two matching solenoids are 4 and 7.5 $\mathrm{kG}$. There is about 1 meter distance between these two solenoids for the ion injection and diagnostics.

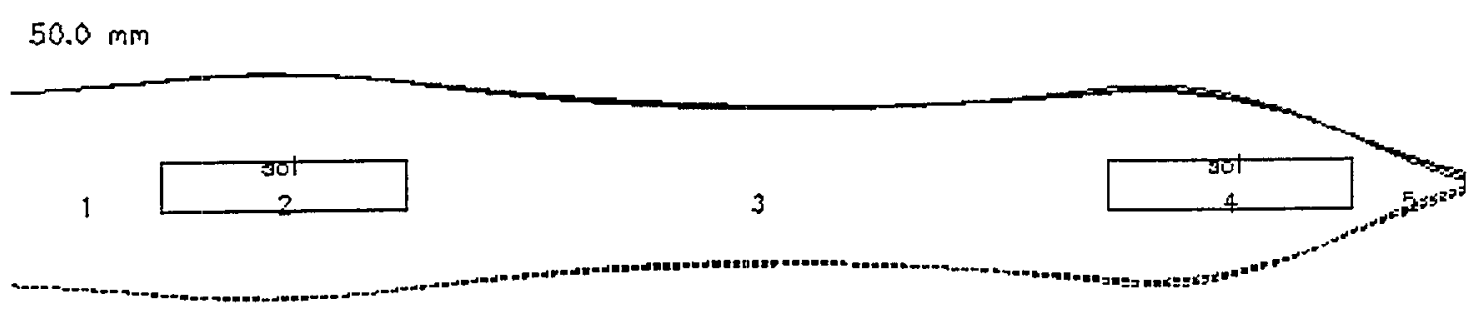

FIGURE 6. TRACE output showing beam transport from EBIS to RFQ

These calculations have been done including the effects of the space charge at the full beam current of $8.5 \mathrm{~mA}$ (all charge states), since the pulse length is too short for neutralization to occur. If the charge-state distribution were broader, as in an ECR or LIS, the space charge from a much higher total beam current would present problems in matching. Calculations have also been done where the beam is matched into the RFQ with electrostatic lenses, and this is still a viable option that is being considered.

\section{RFQ}

The EBIS output energy is $8.5 \mathrm{keV} / \mathrm{amu}$. The RFQ output energy is $300 \mathrm{keV} / \mathrm{amu}$, which is a comfortable input energy for the IH structure. The frequency chosen is 101.28 MHz . The focusing force in the RFQ is proportional to $1 /$ (rf wave length) $)^{2}$, and this frequency provides a good focusing for a relatively low $\mathrm{q} / \mathrm{m}$ beam. Another consideration has been the fact that there are several existing RFQs and IH Linacs operating at this frequency. The emittance from the EBIS source has not yet been fully determined, but based on the available data we estimate it to be about $0.35 \pi \mathrm{mm}$ mrad (normalized, 90\%). The acceptance of the RFQ in the present design is comfortable, at $1.7 \pi \mathrm{mm}$ mrad (normalized), with an aperture radius of $6 \mathrm{~mm}$. 
At a frequency of $101.28 \mathrm{MHz}$, a four rod RFQ can be easily designed and built, and would be very similar to the CERN heavy ion RFQ. Table 5 shows some parameters and specifications of this RFQ.

Fig. 7 shows the calculated transmission as a function of input emittance. There is essentially no calculated emittance growth. Fig. 8 shows the calculated transmission as the input energy is varied. This shows that the RFQ transmission will remain good even if the EBIS beam energy spread is increased (e.g. if the voltage in the EBIS trap is ramped to decrease the ion pulse width). The RFQ transmission remains $>80 \%$ even for currents in excess of $35 \mathrm{~mA}$. Simulations also show that transmission is high for charge states neighboring the desired charge state, that is, the RFQ will not act as a good filter for the off-charge states.

TABLE 5. Parameters of the proposed RFQ

\begin{tabular}{|l|c|c|}
\hline \multicolumn{1}{|c|}{ Parameters } & Value & Units \\
\hline Type & 4-rod & \\
\hline q/m & $0.16-0.5$ & \\
\hline Input Energy & 8.5 & $\mathrm{keV} / \mathrm{amu}$ \\
\hline Output Energy & 300 & $\mathrm{keV} / \mathrm{amu}$ \\
\hline Frequency & 101.28 & $\mathrm{MHz}$ \\
\hline Max rep rate & 10 & $\mathrm{~Hz}$ \\
\hline Length & 2.96 & meters \\
\hline Number of cells & 236 & \\
\hline Aperture Radius & 0.006 & meters \\
\hline Voltage & 92 & $\mathrm{kV}$ \\
\hline E(surface) & 20.8 & $\mathrm{MV} / \mathrm{m}$ \\
\hline Acceptance & 1.7 & $\pi \mathrm{mm}$ mrad (nor) \\
\hline Input Emittance & 0.35 & $\pi \mathrm{mm} \mathrm{mrad,} \mathrm{nor,} 90 \%$ \\
\hline Output Emittance (trans) & 0.375 & $\pi \mathrm{mm} \mathrm{mrad}$, nor, $90 \%$ \\
\hline Output Emittance (longit) & 0.75 & $\pi \mathrm{MeV}$ deg \\
\hline Transmission & 97 & $\%$ \\
\hline Bravery factor & 1.8 & Kilpatrick \\
\hline
\end{tabular}

\section{Medium Energy Beam Transport}

The purpose of the Medium Energy Beam Transport (MEBT) is to match the beam from the RFQ to the IH structure in all three planes (two transverse, and longitudinal). The RFQ has a FODO lattice with $1 \beta \lambda$ period and IH structure has triplet focusing effectively with greater than $6 \beta \lambda$ period. The RFQ and the IH structure have the same $\mathrm{RF}$ frequency of $101.28 \mathrm{MHz}$.

The MEBT requires 6 controllable elements to match the beam in all three planes, four in the transverse plane and two in the longitudinal plane. Because the RFQ is a strong focusing structure, the beam from the RFQ is highly divergent in one transverse plane and highly convergent in the other transverse plane. After passing the high energy RFQ endflange, gate valve, and a current transformer, the beam usually becomes divergent in the both planes by the time it reaches the first quadrupole. To 


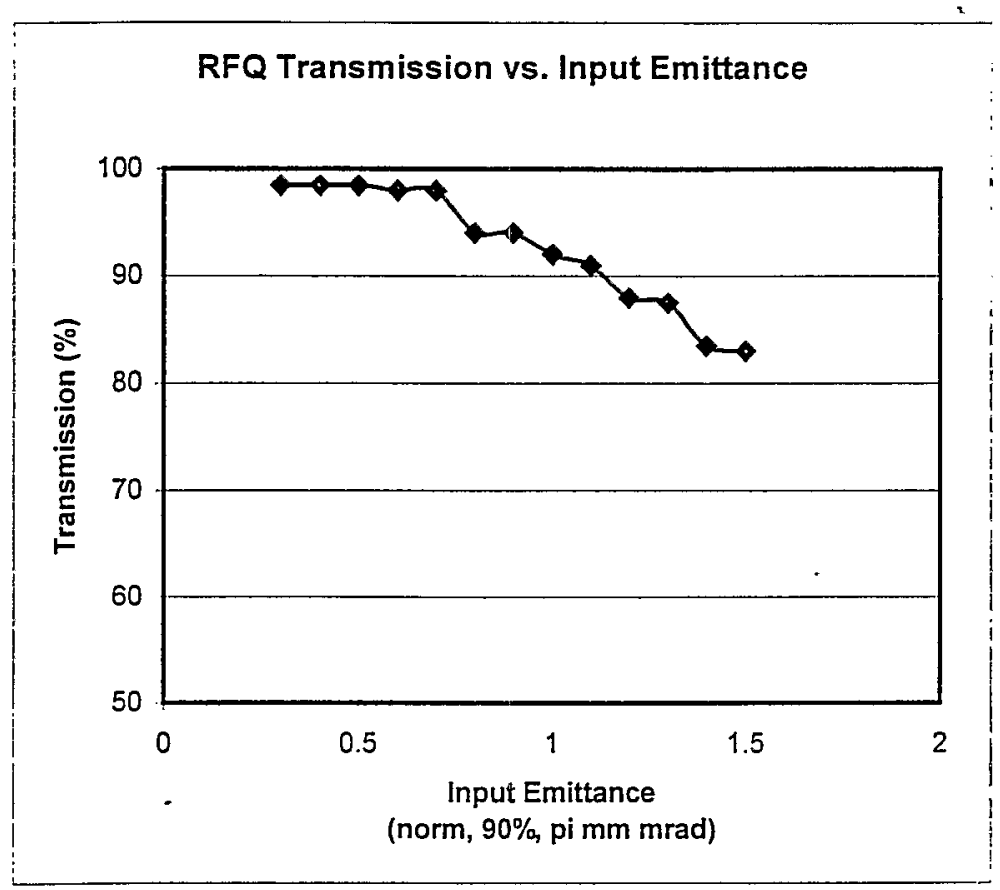

FIGURE 7. Calculated RFQ transmission vs. input emittance.

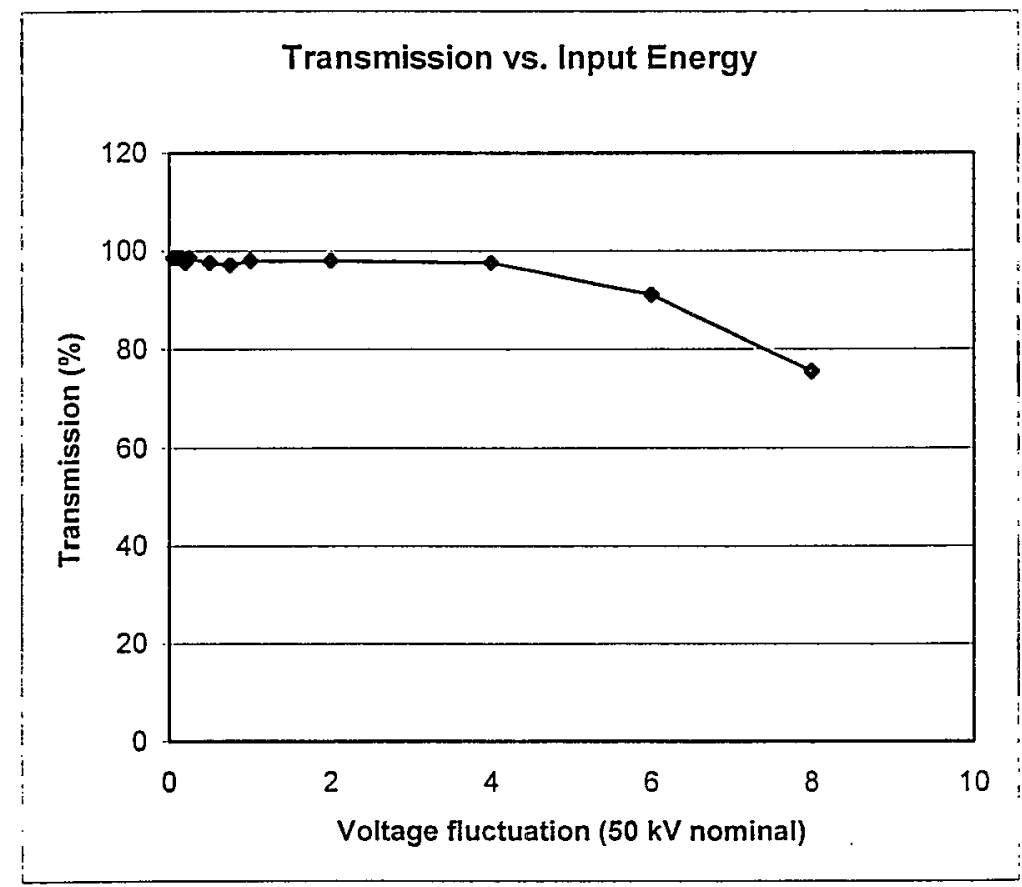

FIGURE 8. Calculated RFQ transmission vs. fluctuation of input energy.

avoid this problem we are using a small permanent magnet quadrupole placed in the high-energy end flange of the RFQ. (This technique has been employed in our proton RFQ). There are four more quadrupoles to provide four degrees of freedom in the transverse plane. In the longitudinal plane we use one buncher and the position of the buncher to match the beam to the $\mathrm{IH}$ structure. Thus we have 5 active controls to match beam instead of six. Figure 9 shows the TRACE3D output for the MEBT. 


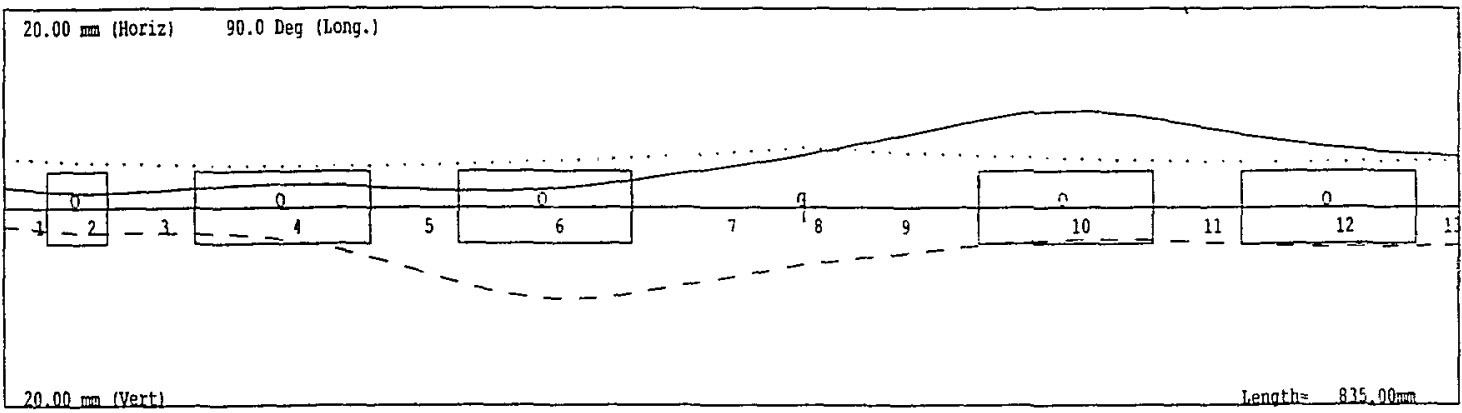

FIGURE 9. TRACE3D output showing the MEBT horizontal and vertical beam envelopes.

\section{IH Linac}

For the baseline design, we have chosen an Interdigital-H (IH) structure, as has been used at CERN for the $\mathrm{Pb}$ Linac, and at GSI. This will be a single-cavity, designed for a fixed output velocity, independent of the $\mathrm{q} / \mathrm{m}$ of the desired beam (cavity gradient is adjusted for different $\mathrm{q} / \mathrm{m}$ 's, to maintain a fixed velocity profile). In the first year of the project, we will continue to investigate alternative structures, including independently phased superconducting cavities, similar to the ATLAS structure at Argonne, or the room temperature structure used for the TRIUMF rare-ion accelerator. Either of these last two would offer the potential advantage of allowing acceleration to higher energies for higher $\mathrm{q} / \mathrm{m}$ ions.

As mentioned previously, we have selected a final energy of $2 \mathrm{MeV} / \mathrm{amu}$ into the AGS booster, to reduce the space charge tune shift and the electron capture cross section at injection. The Linac frequency is chosen to be $101.28 \mathrm{MHz}$, which is the same as the CERN Pb IH Linac. This design is an extension of the CERN Pb Linac in which the beam dynamics concept of "combined zero degree synchronous particle sections" is used.

The Linac has one tank, 4 meters long, with two quadrupole triplets inside for focusing. The maximum field on the axis will be $13.5 \mathrm{MV} / \mathrm{m}$. The gap voltage distribution is adjusted by changing the capacity distribution between the adjacent drift tubes to match the velocity profile. Table 6 shows the main parameters of the IH Linac. The computer code LORAS [4] has been used to design and simulate the IH Linac.

TABLE 6. Main parameters of the proposed IH Linac

\begin{tabular}{|l|c|c|}
\hline \multicolumn{1}{|c|}{ Parameters } & Value & Units \\
\hline $\mathrm{g} / \mathrm{m}$ & $0.16-0.5$ & \\
\hline Input energy & 0.300 & $\mathrm{MeV} / \mathrm{amu}$ \\
\hline Output Energy & 2.0 & $\mathrm{MeV} / \mathrm{amu}$ \\
\hline Frequency & 101.28 & $\mathrm{MHz}$ \\
\hline Max rep rate & 10 & $\mathrm{~Hz}$ \\
\hline Length & 4.0 & $\mathrm{~m}$ \\
\hline Input emittance & 0.55 & $\pi \mathrm{mm} \mathrm{mrad}, \mathrm{norm}, 90 \%$ \\
\hline Output emittance & 0.61 & $\pi \mathrm{mm} \mathrm{mrad}$, norm, $90 \%$ \\
\hline Output energy spread & 20.0 & $\mathrm{keV} / \mathrm{amu}$ \\
\hline Transmission & 100 & $\%$ \\
\hline
\end{tabular}




\section{High Energy Beam Transport}

The High Energy Beam Transport (HEBT) matches beam transversely from the Linac to Booster injection, minimizes the energy spread at the injection, provides ion charge state discrimination, and provides space for diagnostics. It uses as much as possible the existing components of the heavy ion transfer Linac from Tandem to Booster. A preinjector location is being considered in which the HEBT joins the existing Tandem-to-Booster (TTB) transfer line at the first of two existing 13 degree dipoles. Figure 10 shows the TRACE3D calculation for transport of the beam from the Linac to Booster.

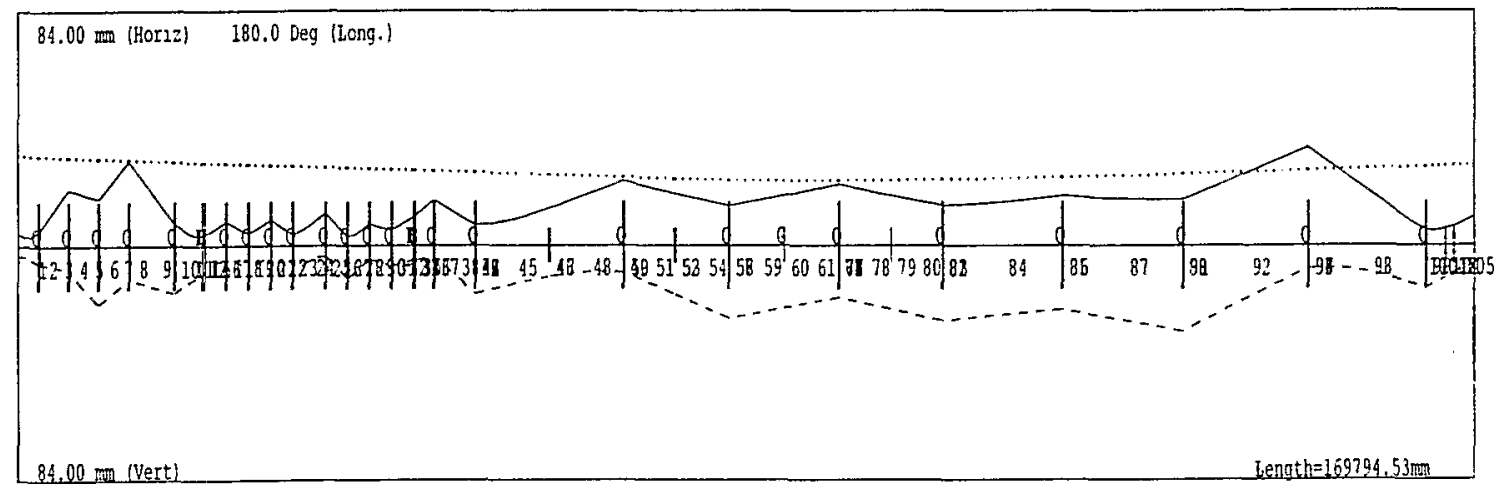

FIGURE 10. TRACE3D calculation of horizontal and vertical beam envelopes from Linac to Booster.

Functionally, we can divide the line into three sections (1) matching from Linac to transport, (2) achromat and charge discrimination, and (3) matching into the Booster (including debuncher):

1. Matching from the Linac: This section has five quadrupoles to match the smaller beam from the Linac triplet focusing into the transport FODO lattice. After the fourth quadrupole there is a 15 foot drift through the wall between the two tunnels, which disrupts the FODO regularity, so the beam size is relatively large in this region.

2. Charge discrimination: The RFQ and Linac will not eliminate all unwanted charge states. Therefore, for charge discrimination we use the existing section of the TTB line which has two 13 degree dipole magnets. Between these two dipoles we have 8 quadrupoles to make this section achromatic. For gold, charge 32 and 33 will separate by $6.6 \mathrm{mrad}$ after the first dipole magnet. The undesired charge can easily be collected after the first quadrupole in the achromat on slits, since at this slit location the separation between these charges will be about $48 \mathrm{~mm}$.

3. Matching into Booster: A debuncher cavity is located at 80.3 meters from the Booster injection, and rotates the longitudinal phase space to minimize the energy spread at injection. The final transverse matching into Booster is done with four quadrupoles. 


\section{BOOSTER INJECTION WITH THE EBIS BEAM}

The expected parameters of the EBIS beam at the Booster are presented in Table 7. The pulse width will allow for 1 to 4 turn injection at the Booster. It may also be possible to extract ions from the source in short bursts to match directly the Booster if buckets. With the Booster RF harmonic number 4 or 6 , this would require a bunch length of about $1.23 \mu s$ or $0.83 \mu s$, respectively.

The beam energy spread at the exit of the Linac is $\pm 20 \mathrm{keV} / \mathrm{u}$, which is reduced by a debuncher to $\pm 2 \mathrm{keV} / \mathrm{u}$. This is crucial for keeping the Booster beam longitudinal emittance small.

TABLE 7. Expected beam parameters at Booster injection

\begin{tabular}{|c|c|c|c|}
\hline$A u^{32+}$ & & & Unit \\
\hline Particles per pulse & $N$ & 3 & $10^{9}$ \\
\hline Kinetic energy & $E_{k}$ & 2 & $\mathrm{MeV} / u$ \\
\hline & $\beta$ & 0.0652 & \\
\hline & $\gamma$ & 1.002 & \\
\hline & $d$ & $10-40$ & $\mu \omega$ \\
\hline Pulse width & $\Delta E$ & \pm 2 & $\mathrm{keV} / \mathrm{u}$ \\
\hline Energy spread & $\Delta p / p$ & \pm 0.05 & $\%$ \\
\hline Momentum spread & $\Delta p / 7$ & $\pi \mathrm{mm} \mathrm{mrad}$ \\
\hline Norm. 95\% emittance & $\varepsilon_{N .95}$ & 0.7 &
\end{tabular}

\section{Space Charge Effect}

The space charge effect is estimated by the incoherent tune spread

$$
\Delta v_{\text {inc }}=\frac{-3 N Q r_{0}}{2 \pi A B_{f} \beta \gamma^{2} \varepsilon_{N, 95}}
$$

where $Q$ and $A$ are the charge state and atomic mass, respectively, $r_{0}$ is the classical radius of proton, and $B_{f}$ is the bunching factor. The bunching factor at early acceleration is considered to be $\leq 0.4$. With 1 to 4 turn injection, the incoherent tune spread is calculated as 0.62 to 0.16 .

Present Booster beam with the Tandem injection of 40 turns usually has normalized horizontal and vertical $95 \%$ emittances of 6 and $3 \pi \mathrm{mm} \mathrm{mrad}$, or 8 and 4 times larger than the EBIS beam. Therefore, the Booster beam transverse emittance with the EBIS injection will not be a problem. In fact, emittance smaller than with Tandem beam injection can be expected, which may have a positive impact on the RHIC luminosity.

\section{Capture of Unbunched Beam}

The RF capture and the resultant longitudinal beam emittance are important issues. The present optimized scheme for the Tandem beam injection is to use an RF voltage of about $200 \mathrm{~V}$, with harmonic number of 6 , at the $\dot{B}=0$ porch to capture the beam. The adiabatic-like capture takes about $6 \mathrm{~ms}$. The resultant longitudinal emittance is 
$\sim 0.05 \mathrm{eVs} / \mathrm{u}$ for the one Booster pulse ( 6 bunches). Once this beam becomes a RHIC bunch, the longitudinal emittance is enlarged to 0.3 to $0.4 \mathrm{eVs} / u$. Therefore, with the EBIS beam injection, the resultant Booster pulse longitudinal emittance should be not larger than $0.05 \mathrm{eVs} / \mathrm{u}$.

The capture loss needs to be as small as possible. It is known that the lost beam may create a pressure increase, causing further beam loss. The injected beam momentum spread will have an impact on both aspects. Note that the Tandem beam momentum spread is very small, at about $\Delta p / p= \pm 0.01 \%$. With the EBIS beam momentum spread of $\Delta p / p= \pm 0.05 \%$ at the Booster injection, and much fewer turns injected, the injection scheme is substantially different from the existing one.

Simulations have been done for different schemes of the EBIS beam capture. It can be observed that all particles can be captured, and also the resultant emittance is relatively small. In fact, there is almost no longitudinal emittance growth in the capture. For a total of 6 buckets, the area is calculated to be $0.05 \mathrm{eVs} / \mathrm{u}$.

\section{CONCLUSION}

Brookhaven was very fortunate to have had a Tandem Van de Graaff accelerator, which was upgraded to serve as a preinjector for a heavy ion program at the AGS beginning in 1986, and now is serving admirably as the preinjector for RHIC. Indeed. until the recent progress in EBIS development, there has not been an attractive alternative to the Tandem preinjector. It is now clear that an EBIS source injecting into a Linac-based preinjector can reach the pulsed $\mathrm{Au}$ beam intensity required for RHIC, and offers significant advantages in meeting long-term requirements for performance and reliability for the RHIC program. While continual progress is being made on EBIS development at BNL, we are preparing to request funding to begin fabrication of the new preinjector, over a 4-5 year time period.

\section{ACKNOWLEDGEMENTS}

We would like to thank Dr. Bernhard Schlitt, from GSI, for his help in teaching us how to run the LORAS code.

This work was performed under the auspices of the U.S. Department of Energy.

\section{REFERENCES}

1. Pikin, A., Alessi, J., Beebe, E., Kponou, A., Prelec, K., Kuznetsov, G., and Tiunov, M, "EBTS: Design And Experimental Study", these proceedings.

2. Kponou, A., Beebe, E., Pikin, A., Kuznetsov, G., Batazova, M., and Tiunov, M., Rev. Sci. Instrum. 69, 1120 (1998).

3. Crandall, K.R., and Rusthoi, D.P." "TRACE 3-D Documentation", LANL Report LA-UR-97-886.

4. Ratzinger, U. and Mimje, V.T. "LORAS", description and manual. 